\title{
Blockchain Technology in Pharmaceutical Industry to Prevent Counterfeit Drugs
}

\author{
ljazul Haq \\ International School of Software \\ Wuhan University \\ Wuhan, China
}

\author{
Olivier Muselemu Esuka \\ International School of Software \\ Wuhan University \\ Wuhan, China
}

\begin{abstract}
The production and distribution of counterfeit drugs is an urgent and increasingly critical worldwide issue, especially in developing countries. The market value of pharmaceutical counterfeiting has reached billions of dollars annually. One of the reasons for drugs counterfeiting is the imperfect supply chain system in pharmaceutical industry. Drugs change ownership from manufacturers to wholesaler, distributer and then pharmacist before it reach the customer. In current supply chain system, information is not shared between systems, manufacturers don't know what happened to their products, drugs regulatory authority has no visibility of the system, recalls are complicated and costly, and companies cannot follow-up patients. In this paper we explain how to use blockchain technology in pharmaceutical supply chain to add traceability, visibility and security to the drugs supply system. The proposed system will be used in pharmaceutical industry to track the drugs from its manufacturing until its delivery to patient. After the usage of a drug, its effect on patient will be recorded to a database for future statistics. A permissioned blockchain will be used for storing transactions and only trusted parties will be allowed to join the network and push data to blockchain.
\end{abstract}

\section{Keywords}

Blockchain, Information Security, Counterfeiting, Pharmaceutical Supply Chain.

\section{INTRODUCTION}

Pharmaceutical Research \& Development is a complex process that takes several years from drug discovery to drug development and regulatory approval. When all the process is done and a standard product is developed, the next challenge for manufacturers is to deliver the product to the intended customer in its original form and to ensure that the customer get the genuine product that is developed by the legitimate manufacturer, not by counterfeiter. But the current Supply Chain Management (SCM) system of pharmaceutical industry is outdated, and doesn't provide visibility and control for manufacturers and regulatory authority over drugs distribution and it cannot withstand the $21^{\text {st }}$ century cyber-security threats. This situation of SCM leads to the production, distribution, and consumption of counterfeit drugs. Counterfeit drugs have created a particularly dangerous public health risk and increasingly acute worldwide issue especially in developing countries. These counterfeit drugs directly and indirectly adversely affect health. Indirectly, these drugs do not contain the dosage or active agent required to kill the disease, that finally

cause drug-resistant strains, and then even using the original drugs are useless. More directly, such counterfeits may contain active ingredients, but the amount is too low or too high, or produced in an impure manner that contain toxic ingredients, in this case it can cause very serious health problems [1]. Counterfeit drugs manufacturers sometimes use the brand logo of legitimate manufacturers and make fake products used in daily life, that's less harmful. But in many cases they affect the drugs for the treatment of cancer, painkillers, cardiovascular disorders, antibiotics, contraceptives and other prescription drugs, that can lead to very serious results [2]. Estimates of the numbers of counterfeit pharmaceuticals range from 10 to 15 percent of the world drug supply. $30 \%$ of the drugs sold in developing countries are counterfeit [2]. WHO estimates that of the 1 million deaths that occur each year due to malaria, 0.2 million of them are the result of counterfeit anti-malarial drugs [3]. Counterfeit drugs for tuberculosis and malaria kill 0.7 million people every year [1]. Counterfeiting is perhaps one of the oldest and most lucrative businesses, but the advancement in technology have much facilitated the business of counterfeiters [4], that's why FBI calls counterfeiting the crime of the $21 \mathrm{st}$ century [5], because production and distribution of counterfeited goods is increased, and they can be produced in a large quantity in short amount of time. According to the International AntiCounterfeiting Coalition (IACC), counterfeiting has become one of world's largest and fast growing criminal business, with an estimated value of more than US\$ 600 billion annually [6].

For the prevention of counterfeit drugs, pharmaceutical industry need an efficient supply chain management system, and the best available solution to develop a perfect SCM system is the Blockchain technology. Blockchain is a distribute ledger system (firstly introduced by a pseudonym Satoshi Nakamoto in 2008 [7]) that has shown widespread adaptability in recent years and a variety of market sectors sought ways of incorporating its abilities into their operations. Although, so far most of the focus has been on the financial services industry, but now projects in other service related areas, such as healthcare, energy and legal firms also started using this marvel. Supply chain security is one aspect that has recently won attention. Any product subject to a sensitive production process and widespread reputational issues are associated with the final product, the benefits of Blockchain are evident. Blockchain is the best fit in those scenarios where privacy protection and data security is the highest priority. 
Therefor pharmaceutical supply chain present a further use case of Blockchain technology.

This paper is organized as follows: In section 2 we have discussed the work of other researchers and startups related to this issue. The purpose of the proposed system and the need of blockchain is explained in section 4 . Implementation of the system is discussed in section 5 and the paper is concluded in section 6 .

\section{RELATED WORK}

Blockchain technology initially became popular for the realization of cryptocurrency (i.e. Bitcoins) and other financial services. Later on, a lot of blockchain applications suggested in various fields, and after the introduction of smart contracts, blockchain became more powerful. With the enormous adaptability of Blockchain, several ideas have been proposed, in order to incorporate its features in the field of medicine and healthcare. Benchoufi and Ravaud [8] has explained the use of blockchain for improving the clinical research quality. They have discussed overall the use of blockchain in healthcare and medicine but no explanation is given about the use of blockchain in supply chain of drugs. Healthcare data is very valuable and is prone to various types of attacks, MedShare is another proposal that aim to use blockchain technology in health care to share medical data from one entity to another in a trustless environment [9]. MedRec, a white paper published that introduce a system for storing secrete medical data and to provide it for study purpose in future to researchers. It presents a system for storing patient's data and to easily access that data by incorporating blockchain security [10]. M. Mettler has also mentioned the use of blockchain in pharmaceutical supply chain but has lack of implementation detail [11]. Besides that, several articles and academic journals have been published on this issue, interested readers are suggested to read [12] [13] [14].

\section{PURPOSE}

While looking at the issues mentioned in section I, we realized that the pharmaceutical industry need an updated supply chain system. The purpose of the new system is to incorporate the features of blockchain technology and add traceability, and security to the drugs supply chain, and to provide visibility to manufacturers and drugs regulatory authority of the SCM system. In such scenarios where we need data privacy and data accessibility both at the same time - blockchain technology is the best choice. Every time a product changes hands, the transaction can be documented to create a permanent history of a product, from manufacture to sale. This will dramatically reduce time delays, costs, and human error that occurs in transactions today. The purpose and features of the blockchain based SCM system for pharmaceutical industry are summarized as follows:

- To Increase Trust and Transparency - With manufacturer and customers being able to track pharmaceutical products throughout the supply chain, they will trust each other. Manufacturers will be able to see that the products they want to deliver is safely received by the intended client. On the other hand, client will be able to see that the product he want to buy is developed by a legitimate manufacturer, and he got it in its original form.

- Traceability - Once the manufacturer produce a product he will register it on the blockchain, and here after the drugs will be tracked, traced and authenticated at each stage of their journey. As the drugs ownership change physically, its ownership will be transferred simultaneously on the blockchain network. Drugs manufacturers will be able to see the journey of their products at any of time, from manufacturing to packagers, and from packagers to distributers.

- Add Visibility and Protect Privacy: Visibility and privacy are mostly opposite to each other and to obtain one we often lose the other. Blockchain is the best technology for the trade-off that can guaranty to verify the originality of a piece of data that is made available publically while keeping the private data of an entity secrete and without any compromise on privacy. In a pharmaceutical supply chain system, the products will be verifiable without any information about the manufacturer's secrete techniques. On the other hand, the patient's medical record will be accessible to different participants on the network - without knowing the private data of the patient.

- Extended Security - Blockchain is considered as one of the most secured ledger systems on the planet. Blockchain is an immutable database and the information once stored on it, it cannot be deleted or modified. In the proposed system, a permissioned blockchain will be used that is more secure then the public blockchain, in which only legitimate participants will be granted privileges to push data to the blockchain.

- Database for Future Statistics - The effect of drugs on patient will be recorded, that record will be handful for doctor to suggest dose to a patient in future. Using conventional databases, this type of record keeping was not secure, and patient's privacy was at risk, but using blockchain, patient's data can be stored without sharing his private record.

\subsection{Why Blockchain?}

Blockchain is a distributed network of computers that share a secure ledger of transactions among the participants connected to the network without any central server. It stores every transaction occurs in the network with a timestamp and eliminate the need of third party. Every node on the network maintains a local copy of the ledger, and any small change in the local ledger is replicated to the overall network, and every node on the network update their local ledger. If one node is failed or disconnected, it doesn't have any effect on the network. The first reason to use Blockchain technology in such a system like pharmaceutical SCM is its security. Blockchain is the best option to provide $21^{\text {st }}$ century cyber-security, and until now there is no breach found in it. It is designed to prevent any single person from modifying the data and transactions, this way blockchain can increase the trust and help to eliminate the biasness found in traditional supply chain systems. Using blockchain, participants can anonymously exchange digital assets, where they don't need to know each other and trust each other or a third participant for their transaction. Therefor blockchain is the best option to transfer trust in a trustless world. One of the reasons we suggest blockchain for pharmaceutical SCM system is that it's the best option to record the journey of a product across supply chain. Every time the product change ownership, a new transactions will pushed to blockchain. Storing the history of a product make it easy to reveal its actual origin and milestones. This approach will bring more transparency to transactions in the pharmaceutical supply chain. 
The power of blockchain is not only limited to the creation of cryptocurrency but any type of digital asset can be created and traced. In the context of pharmaceutical supply chain, when a manufacturer create a product and register it on the network, it will become a digital asset, and could be transferred to other participant just like cryptocurrency. For privacy protection, blockchain is again in the first row. In a blockchain network, every participant is assigned a key-pair ${ }^{1}$ as identifier. The private data of a participant is kept secret and every transaction is done from the public key of one participant to the public key of the other. In pharmaceutical SCM system, the private data of patients will be kept secret and their medical record will be used public - anonymously.

The last and the most important reason of using blockchain in pharmaceutical SCM system is the Smart contract. A smart contract is a piece of code that contain the actual rights and obligation that include the term and conditions for the payment and delivery of goods and services agreed upon by all the signees and can be automatically executed. Smart contract can add greater intelligence and more power to blockchain. They can be used to make state-of-the-art and cutting edge customized blockchain based systems.

\section{IMPLEMENTATION}

To incorporate blockchain technology in pharmaceutical supply chain system, we should first understand how blockchain ledger works under the hood. Blockchain has a built-in identity mechanism, a cryptographically secure key pair (as mentioned in the above section). These keys are used to assign each participant a specific activity on the network. A participant can be a device, person or an entity. The original identities of participants are hidden and they are known by these keys. A key pair contains no clue about the participant, but additional information (e.g. name, contact or professional credentials) can be associated with it [15]. But the best approach is to keep these additional information off-chain and merge them with on-chain data (key pair) using there IDs. In the context of pharmaceutical supply chain management, the participants will be the manufacturer, packager, distributer and doctor etc. Each of these participants will be identified by their unique key pair on the network. Drugs will be considered as the assets, with each of them having a unique key (or hash). The ID will be attached with drug in the form of QR Code.

While keeping in mind the basic architecture, the proposed system can be implemented on different ways depending on one's preferences. A lot of third party APIs are also available that can be used to push the data and transactions to blockchain network, a few of them are here [13] [16] [17]. Each of these APIs provide different types of services. Regardless of which programming language or API we use, the basic architecture of our system will be the same.

The selection of a specific blockchain network for storing transactions is also a crucial part, but before that, we should know the types of blockchain. Blockchain has two main types Public blockchain and Permissioned (or private) blockchain, a detail is given here [18] [19]. In a permissioned blockchain not everyone can write to blockchain, only those participants who

\footnotetext{
${ }^{1}$ A combination of cryptographically generated two alphanumeric keys, one is private the other is public
}

has given access, can write or access information on the blockchain. In the context of pharmaceutical supply chain - the better option is to use a permissioned blockchain. The next step is to use a specific blockchain network to save the transactions record, but it totally depends on the developer's choice. Few types of blockchain networks are available in the market now, e.g. Bitcoin Blockchain [20] that is the pioneer one, Ethereum [21], Hyperledger [22] or even BigchainDB [23] can also be used. But the one we suggest is permissioned Ethereum blockchain.

\section{HOW IT WORKS?}

In this section we will discuss how a blockchain based pharmaceutical supply chain management system will work. Let say we have setup a secured and trusted network, where only the trusted parties are given permission to join the network. On the backend there is a permissioned blockchain to store all the required transactions, and once the information entered to it can never be changed. Besides that we have a user-friendly mobile APP that the participants will use to make transactions to the blockchain.

When a factory produce a new product, they will create a unique hash and assign it to the product. The product will be registered on the blockchain using its hash (unique ID). The product will be considered as a digital asset on the blockchain network, and its hash will be used to track it any time on the network. Any additional information of the product can be stored off-chain or on-chain depends on manufacturer's choice. Off-chain data will be merged with on-chain data by using some kind of identifier. Conventionally, in most blockchain based applications a hashdigest (e.g. SHA-256) of all the off-chain data is generated and linked it to the on-chain data. But the best approach is to store large files (e.g. images) off-chain and text data on-chain. Once the product is registered to the blockchain by the manufacturer, its ownership will be easily transfer to another participant using a user-friendly mobile app. Let say the wholesaler want to purchase the drugs from the manufacturer, manufacturer will physically transfer the drugs to the wholesaler and a transfer transaction will be registered to the blockchain simultaneously. Wholesaler will repeat the same process to transfer the drugs to distributer, and distributer will do the same business with pharmacy. In Figure 1 the basic structure of a blolkchain based pharmmacutical chain management is given.

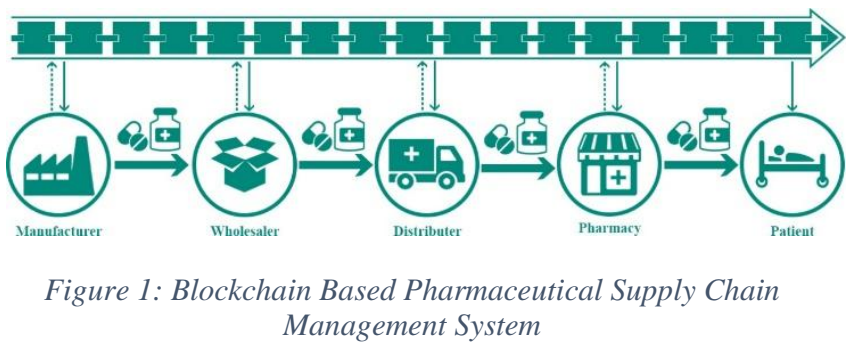

Now let's consider Doctor Alice need some drugs and he want to purchase if from a pharmacy. Using the mobile app, Dr. Alice will first query for drug's ID, to confirm all of its journey from manufacturer to the pharmacy. If the product is genuine, mobile app will show all of its history and if the drug is counterfeit - no record will be displayed. Once Dr. Alice is sure about the originality of the drugs he will then purchase them. Same as the 
doctor, other participants (e.g. Nurse, Family and Patient etc.) will also be able to track the journey of the drugs. A simple layout of the system's front-end is shown in figure 2.

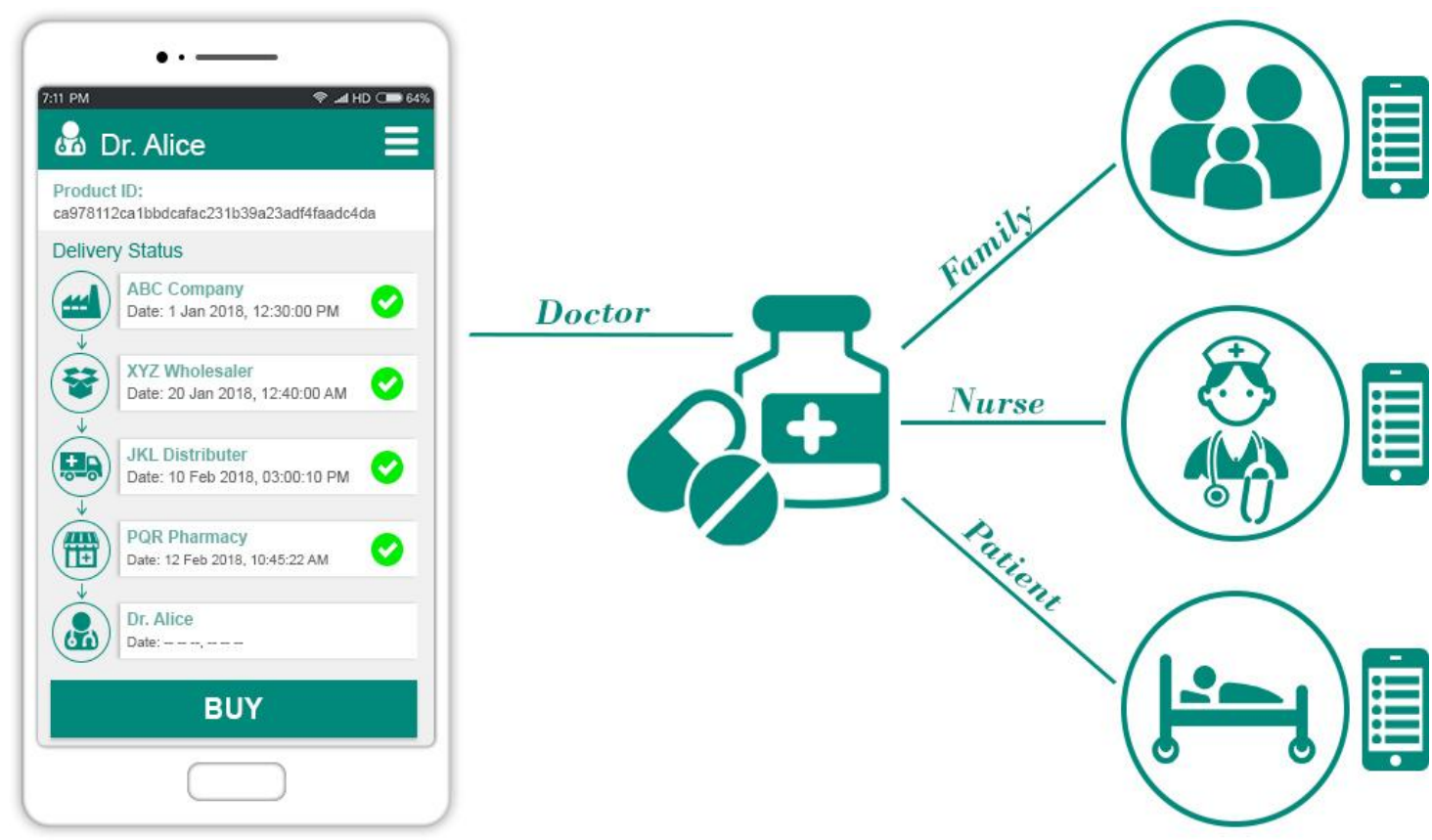

Figure 2: A simple layout of the system's front-end

\section{CONCLUSION}

In this paper, we proposed a further use case of blockchain technology in healthcare. We pointed out the issues in current pharmaceutical supply chain management, and explained how blockchain can be used to add traceability and visibility to drugs supply and overcome the issue of counterfeiting. How the identity mechanism of blockchain works and how is it helpful to share medical data while keeping the patient's private data secrete is explained. We highlight the possible techniques, blockchain types and third party solutions that can be used to implement a blockchain base supply chain for pharmaceuticals. In the last we explained the working of the suggested system with an example that shows how the system will be easily used by different participants.

\section{REFERENCES}

[1] S. F. Roy and M. Jerremy, "African Counterfeit Pharmaceutical Epidemic: The Road Ahead," ACAPPP, 2009.

[2] "WHO | Growing Threat from Counterfeit Medicines," Bulletin of the World Health Organization, vol. 88, no.4, pp, 2010.

[3] H. Julian, S. Philip and M. Julian, "Combating the spread of fake drugs in poor countries," International Policy
Network, 2009.

[4] G. R, "Te state control of medicines: Te frst 3000 years.," Br. J. Clin. Pharmac.,, vol. 8, no. 2, pp. 93-305, 1979.

[5] "United Nations Interregional Crime and Justice Research Institute (UNICRI)," Global counterfeiting:, 2003.

[6] E. Roxanne, D. K. Lisa and P. W. George, "Anticounterfeiting in the fashion and luxury sectors: trends and strategies," Anti-counterfeiting - A Global Guide, 2013.

[7] N. Satoshi, "Bitcoin: A Peer-to-Peer Electronic Cash System," 2008.

[8] . Mehdi, . Raphael and . Philippe, "Blockchain protocols in clinical trials: Transparency and traceability of consent," F1000Research, 2017.

[9] X. QI, B. S. EMMANUEL, O. KWAME, G. JIANBIN, D. XIAOJIANG and G. MOHSEN, "MeDShare: Trust-Less Medical Data Sharing Among Cloud Service Providers via Blockchain," IEEE Access, 2017.

[10] A. Asaph, E. Ariel, V. Thiago and L. Andrew, "MedRec: Using Blockchain for Medical Data Access and Permission Management," in 2nd International Conference on Open and Big Data, Cambridge, MA, 02139, USA, 2016.

[11] M. Mettler, "Blockchain Technology in Healthcare: The Rovolution Starts Here," in IEEE 18th International 
Conference on e-Health Networking, Applications and Services, Healthcom, 2016.

[12] C. Edward, L. Ying, Z. Jia and L. Yang, "Healthcare services across China - on implementing an extensible universally unique patient identifier system," International Journal of Healthcare Management , pp. 1-7, 2017.

[13] "Tierion - Blockchain," [Online]. Available: https://tierion.com/. [Accessed 241 2018].

[14] "Every Product Has a Story," Provenance, [Online]. Available: https://www.provenance.org/. [Accessed 21 2018].

[15] "Blockchain in Healthcare," [Online]. Available: https://www.hyperledger.org/wpcontent/uploads/2016/10/ey-blockchain-in-health.pdf. [Accessed 231 2018].

[16] "Blockcypher," [Online]. Available: https://www.blockcypher.com/. [Accessed 11112018 ].

[17] "BlockRx," iSolve, [Online]. Available: https://www.blockrx.com/. [Accessed 11 2018].

[18] G. Jeff, "Public versus Private Blockchains - Part 1: Permissioned Blockchains," 2015.

[19] G. Jeff, "Public versus Private Blockchain - Part 2: Permissionless Blockchains," 2015.

[20] "Bitcoin," Bitcoin Blockchain, [Online]. Available: https://bitcoin.org/. [Accessed 112 2017].

[21] "Ethereum," Ethereum, [Online]. Available: https://www.ethereum.org/. [Accessed 1411 2017].

[22] "Hyperledger," Linux Foundation, [Online]. Available: https://www.hyperledger.org/. [Accessed 2512 2017].

[23] "BigchainDB," BigchainDB, [Online]. Available: https://www.bigchaindb.com/. [Accessed 201 2018]. 